\title{
Stabilized, short cavity Brillouin ring laser source design for fiber sensing applications
}

\author{
Leonardo Rossi ${ }^{1}$, Filippo Bastianini ${ }^{2}$, and Gabriele Bolognini ${ }^{1, *}$ \\ ${ }^{1}$ Consiglio Nazionale delle Ricerche, IMM Institute, 101 via P. Gobetti, 40129 Bologna Italy \\ ${ }^{2}$ Sestosensor s.r.l., 140 via Gesso, 40069 Zola Predosa, Italy;
}

\begin{abstract}
A new pump-seeded, short-cavity Brillouin ring laser source layout intended for Brillouin sensing applications is showcased, showing increased high maximum output $(1.5 \mathrm{~mW})$, a strong linewidth narrowing effect (producing light with a linewidth of $10 \mathrm{kHz})$ and limited RIN $(\sim-145 \mathrm{~dB} / \mathrm{Hz})$, providing an ultranarrow, highly stable BRL source that can also be employed as a pump-probe source for BOTDA applications.
\end{abstract}

\section{Introduction}

Brillouin Ring Lasers (BRLs) are a fiber-based light source in which a light (defined as seed pump) is injected and circulates inside a closed fiber loop, where it produces a coherent, downshifted and counterpropagating light (defined as Stokes light) through a process known as Stimulated Brillouin Scattering (SBS). Due to their capability to provide a light signal which is coherent and with a stable frequency shift from the light it originated from with limited intensity and phase noise, BRLs have been studied for applications such as fiber-optics gyroscopes [1], microwave phase-modulated generators [2] and LIDAR detection. Another potentially interesting field of application is distributed optical fiber sensing, which has gathered significant attention in recent years due to the interesting properties of optical fibers, such as resistance and flexibility [3]. A particularly interesting distributed optical fiber sensing method is Brillouin optical time-domain analysis (BOTDA) [4-6]. In this sensing scheme, which is also based on SBS, an optical pump amplifies a downshifted, counter-propagating probe along a sensing fiber. The extent of this amplification is related to the pump-probe frequency shift with a relation known as the Brillouin Gain Spectrum (BGS), and the shift at which this amplification is most efficient is known as the Brillouin Frequency Shift (BFS) which is dependent on the temperature and strain of every point in the fiber [7]. The pump and probe lights are usually provided by sources such as Phase locked loop (PLL) schemes or optical sideband generation (OSB) techniques [8], which tend to have strict requirements in terms of laser source quality and hardware components. In this context, the natural frequency shift of BRLs and the simplicity of their structure makes them an interesting alternative for low-complexity BOTDA setups. In [9], an implementation of a BRL source based on a long cavity (2 km) hybrid BRL source was developed and successfully employed in a BOTDA sensing scheme, where it allowed for measurements over up to $10 \mathrm{~km}$ of range with a spatial resolution of $4 \mathrm{~m}$ and a frequency resolution of $0.5 \mathrm{MHz}$. While these results were already viable for some BOTDA applications, the BRL cavity was affected by three issues which limited its performance: low maximum output power, high output linewidth (2 $\mathrm{MHz}$ ) and a high relative intensity noise (RIN) for frequencies below $1 \mathrm{GHz}$, which was caused by mode hopping effects. In this work, a new BRL design is presented to overcome these limitations. Unlike the other design, this one is based on a short cavity ( $\sim 4 \mathrm{~m})$ Brillouin ring laser (SC-BRL) in order to suppress mode hopping and improve linewidth narrowing of the Stokes output. Through accurate tuning of the length of the cavity and self-injection locking techniques, the BRL was made resonant for both the seed pump and the Stokes output. As a result, the new source displayed significantly reduced RIN, greatly reduced linewidth of the Stokes light and higher maximum output power.

\section{Short cavity BRL}

The BRL cavity that is studied in this work is shown in Fig. 1. Through the exits 1 and 2 of an optical circulator (OC1), the seed pump light from a distributed feedback laser ( $\lambda=1555 \mathrm{~nm}, 350 \mathrm{kHz}$ linewidth) is injected inside the ring cavity which, at its core, is composed of a loop of standard single mode optical fiber for telecommunication (SMF-28) with a length below $5 \mathrm{~m}$. The loop is closed by a Directional Coupler (DC1) with a 90/10 coupling ratio. While recirculating inside this cavity, the seed pump is scattered through SBS, producing a counter propagating downshifted Stokes light, a portion of which is extracted through DC1 and employed as output after going through exits 2 and 3 of $\mathrm{OC} 1$ to be employed in the sensing system. It is to note SBS is a process that is polarization dependant and is most efficient when the polarizations of Stokes and the seed pump are aligned. To ensure this is

\footnotetext{
* Corresponding author: bolognini@bo.imm.cnr.it
} 
the case, two polarization controllers ( $\mathrm{PC} 1$ and $\mathrm{PC} 2)$ are placed before and inside the ring.

Compared to [9], the length of the ring cavity was reduced from $2 \mathrm{~km}$ to $5 \mathrm{~m}$. As was mentioned above, one of the sources for the high relative intensity noise of the previous cavity were mode hopping effects. These effects are caused by the fact that the frequency at which a BRL emits light (the dominant frequency) is the cavity resonant mode which is closest to the BFS, and the positions of both these elements can shift due to thermal and vibrational noises. When this happens, a different resonant mode might become the one closest to the BFS, and thus the dominant mode might change. As a result, both frequency and intensity noise are increased as the BRL source "hops" from one mode to another [10]. This effect is more prevalent the lower the spacing between resonant frequencies is and conversely can be suppressed by increasing the spacing of the resonant modes enough that only one mode lies inside the BGS at any point, hence the greatly reduced cavity length presented in this work. The simplest way to achieve this is by reducing the cavity length, which is inversely proportional to the resonant mode spacing, from $2 \mathrm{~km}$ down to a length below $5 \mathrm{~m}$, like the one we present in this work.

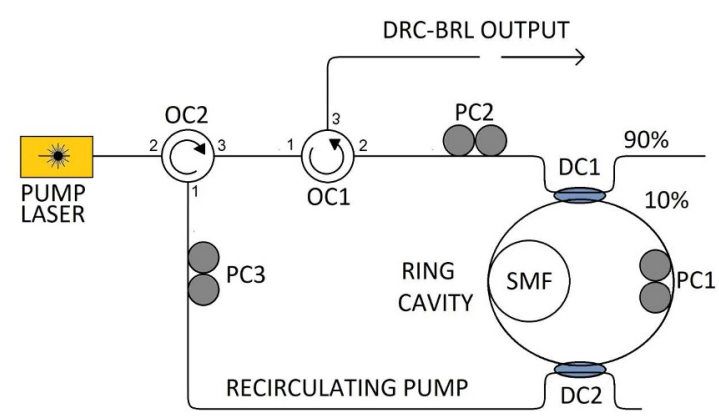

Fig. 1. Scheme of the SC-BRL with self-injection locking.

To counteract the steep reduction in gain medium compared to cavities like the one shown in [9], the cavity is made resonant with both the seed pump and the Stokes output. When this condition, known as double resonance, is satisfied, both lightwaves interfere constructively with newly injected seed pump and newly generated Stokes light at every roundtrip, thus greatly increasing the effective interaction length and overall conversion efficiency.

The resonance of the seed pump is guaranteed through the implementation of a self-injection locking technique in the BRL scheme, which is included in Fig. 1. An optical coupler with a 99/1 coupling ratio (DC2) extracts part of the seed pump inside the ring (which is at the resonant frequency) and sends it back into the DFB laser through exits 1 and 2 of a second optical circulator (OC2). As a result, the DFB is forced to emit at the frequency of the injected light, making the pump resonant with the cavity. In order to make the Stokes light resonant with the cavity, its length is accurately tuned through the use of a single cut technique developed in [11] so that the BFS of the fiber is equal to an integer multiple of the resonant mode spacing. This technique involves measuring the intensity of the Stokes output at different seed pump wavelengths using a tunable laser in place of the DFB, providing a measurement such as the example for a cavity of $5 \mathrm{~m}$ shown in Fig. 2. The goal of this measurement is to locate two consecutive peaks corresponding to wavelengths for which double resonance is satisfied, $\lambda_{L}^{k}$

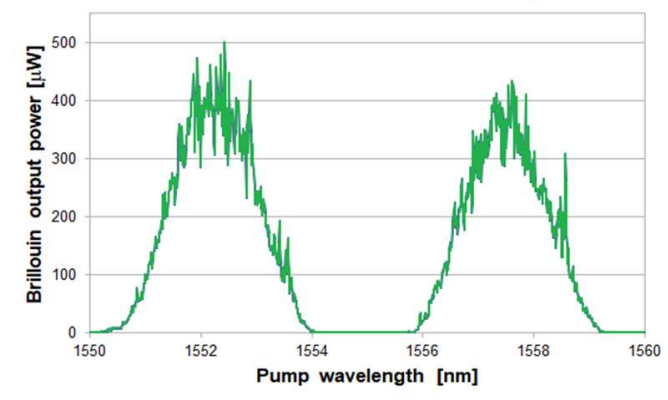

Fig. 2. Double resonance peaks for a cavity length of $5 \mathrm{~m}$.

and $\lambda_{L}^{k-1}$. With these wavelength values, it is possible to estimate the length $\Delta \mathrm{L}$ to be cut from the cavity to move one of those peaks to the desired wavelength $\lambda_{L M}^{k}$ by using the following formula, where $n$ is the refraction index and $V_{a}$ is the fiber's acoustic velocity:

$$
\Delta L=\frac{c \lambda_{L}^{k}}{2 n^{2} V_{a}} \frac{1}{\lambda_{L}^{k-1}-\lambda_{L}^{k}}\left(\lambda_{L M}^{k}-\lambda_{L}^{k}\right)
$$

After the tuning, the cavity was 3.4 meters long.

\section{Performance evaluation}

In order to see how this new design affected the issues noted from the source developed in [6], the Stokes output for this source was evaluated in terms of maximum power, linewidth and intensity noise.

The maximum Stokes output power was found to be $1.5 \mathrm{~mW}$ for a pump power of $25 \mathrm{~mW}$, while the design showcased in [9] was found to provide an output with a maximum power of $0.5 \mathrm{~mW}$ for a $17 \mathrm{~mW}$ pump. The lasing threshold power (the minimum pump power required to obtain Brillouin lasing from the BRL), on the other hand, was $10 \mathrm{~mW}$, up from the $2 \mathrm{~mW}$ found in [9]. These results show that the double resonance effect allows to counteract the greatly reduced amount of gain medium, providing comparable lasing threshold power compared to a long cavity, while providing higher output power, which is a crucial parameter for improved measurement range.

Afterwards, the linewidth of the Stokes output was measured by acquiring the spectrum through the use of the self-heterodyne technique [12], performed with a MachZender interferometer as the one shown in Fig. 3 employing a $150 \mathrm{MHz}$ acousto-optic modulator and a delay line with a length of $12 \mathrm{~km}$ which, when sent into an Electrical Spectrul Analyzer, allows to measure signal spectra with minimum linewidths of $6 \mathrm{kHz}$ [13].

As a result, the output spectrum, shown in Fig. 4, was found to have a full width half-maximum of $10 \mathrm{kHz}$, showing a significant narrowing compared to the output of the long cavity BRL (2.5 MHz), and also significantly narrower than the linewidth of the DFB signal used as 
seed pump (350 kHz), showing the beneficial effect of a short cavity BRL's filtering capabilities.

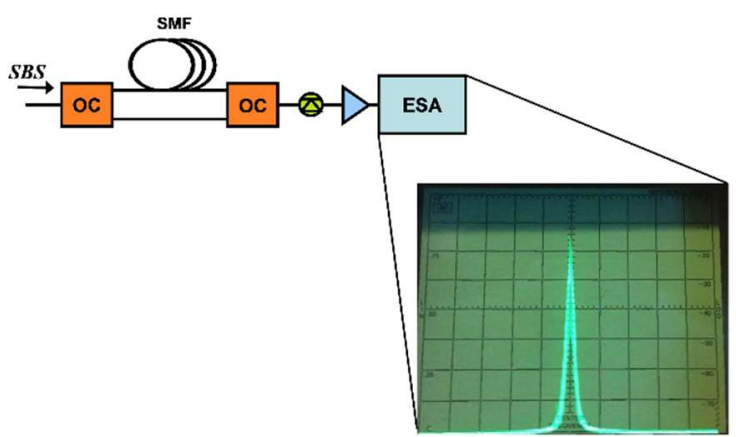

Fig. 3. Self-heterodyne method.

As a further evaluation on self injection locking's capability of suppressing noise effects in BRL, the duration of Stokes lasing intervals with and without selfinjection locking were measured with a photodetector and an oscilloscope. The results are shown in Fig. 2. Without self-injection locking, the duration of Brillouin lasing was found to be between 2 and $10 \mathrm{~ms}$, while with self-injection locking the duration was significantly increased with values ranging from 30 to $90 \mathrm{~ms}$, which is already sufficient for BOTDA measurements. The reason for this difference is of course linked to the seed pump light remaining resonant with the cavity for a much longer time despite thermal and vibrational noise.

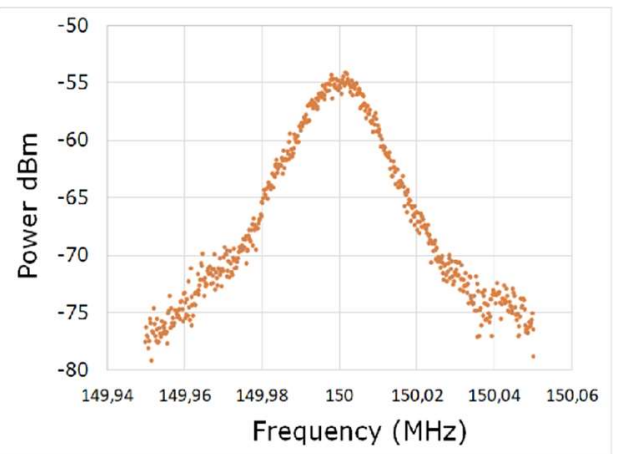

Fig. 4. Short cavity BRL output spectrum.

Finally, in order to evaluate how the employment of a short cavity suppresses noise effects such as mode hopping, RIN measurements were performed in a way similar to [9], as shown in Fig. 6. These measurements were performed by acquiring the BRL Stokes output light with a fast $\mathrm{p}$-i-n photodetector (InGaAs-based, $10 \mathrm{GHz}$ bandwidth) to convert a light signal into a voltage signal, whose power spectrum $\sigma^{2}(\omega)$ in the 1-800 $\mathrm{MHz}$ range was analysed through an ESA (working up to $15 \mathrm{GHz}$ ) after subtracting thermal and shot noise terms due to the photodetector [14]. As is evident from the image, the RIN profile of the SC-BRL ranges between $-140 \mathrm{~dB} / \mathrm{Hz}$ and - $150 \mathrm{~dB} / \mathrm{Hz}$ across the whole $0-800 \mathrm{MHz}$ range, adding a limited amount of intensity noise to the profile of the source DFB. The long-cavity BRL ( $>2 \mathrm{~km}$ length) developed in [9], on the other hand, introduces significant intensity noise, increasing the RIN profile at frequencies below $400 \mathrm{MHz}$, with values up to $-90 \mathrm{~dB} / \mathrm{Hz}$ in the 10 -
$15 \mathrm{MHz}$ frequency range, and becomes similar to the DFB seed pump only beyond $500 \mathrm{MHz}$, where it remains approximately constant with an average value of about - $145 \mathrm{~dB} / \mathrm{Hz}$. From these results it can be easily seen how the reduction of cavity length, mixed with the contribution of self-injection locking suppress noise sources and instability usually associated with fiber cavities that increase the RIN profile of the output compared to the source pump seed [15].

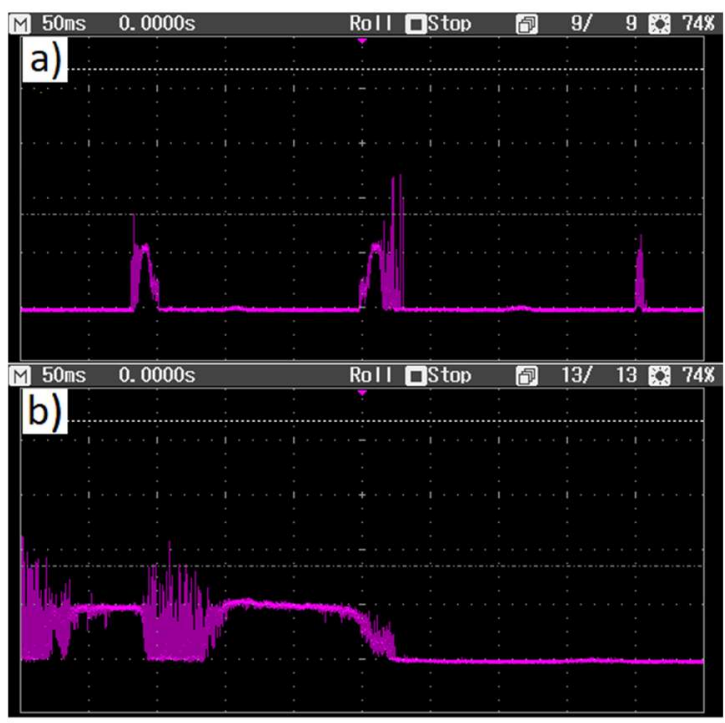

Fig. 5. Stokes output trace without self-injection (a) and with self-injection (b). Timescale: $50 \mathrm{~ms}$ per division.

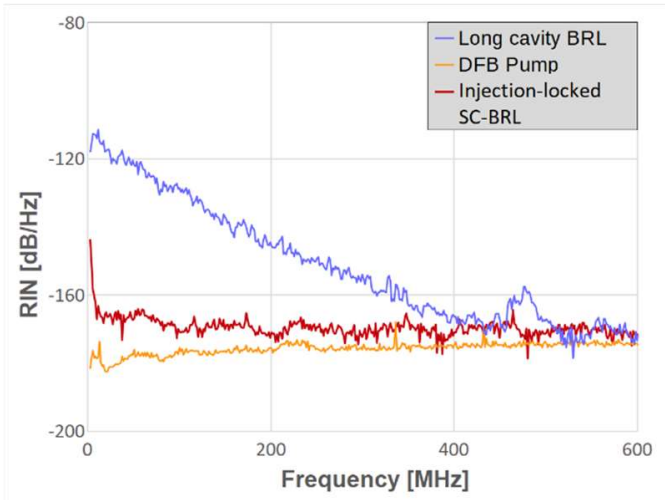

Fig. 6. Comparison of the RIN profiles of the sources.

It can be shown that the reduction in intensity noise provided by the stabilization from self-injection locking and the mode hopping suppression from the employment of the short cavity directly impact BOTDA resolution. To show this, the intensity noise limited signal-to-noise ratio (SNR) for both the SC-BRL source and the long-cavity one was calculated by integrating their RIN spectra over a frequency range equal to the bandwidth of a typical photodiode used in BOTDA (125 MHz). The resulting values were $38.7 \mathrm{~dB}$ for the long cavity BRL and $61.0 \mathrm{~dB}$ for the short cavity one, which implies a SNR improvement of $22 \mathrm{~dB}$. When Lorentzian Curve fitting is used to estract the BFS in BOTDA measurements, source SNR can be translated to frequency resolution $\delta v_{B}$ with the following relation [16]: 


$$
\delta v_{B}=\frac{\Delta v_{B}}{\sqrt{2}(S N R)^{\frac{1}{4}}}
$$

Where $\Delta v_{B}$ is the linewidth of the Brillouin gain spectrum. Due to the linear dependency between the BFS and temperature and strain, their resolutions are also directly proportional to the frequency resolution, and they can be calculated as:

$$
\delta \varepsilon=\frac{\delta v_{B}}{C_{S} v_{B}(0)} \quad \text { and } \quad \delta T=\frac{\delta v_{T}}{C_{T} v_{B}\left(t_{r}\right)}
$$

where $v_{B}(0)$ and $v_{B}\left(t_{r}\right)$ are the Brillouin frequency shifts of unstrained sensing fiber and of sensing fiber at reference temperature, respectively, while $C_{S}$ and $C_{T}$ are the linear temperature and strain coefficients.

From the relations above is immediate to see that, for sources whose SNR is limited by intensity noise, such as BRLs, a $22 \mathrm{~dB}$ improvement is equivalent to a resolution improvement of $5.5 \mathrm{~dB}$ which, converted in linear scale, is equal to an improvement in frequency, strain and temperature resolutions by a factor of $\sim 3.5$.

\section{Conclusion}

In this work, the development and characterization of a Brillouin Ring Laser based on a doubly resonant short cavity to be employed as a source for Brillouin optical time domain analysis were showcased and discussed. Compared to previously developed designs, which used long $(>2 \mathrm{~km})$ cavities, the employment of a short cavity was expected to provide substantial improvements in terms of output linewidth and intensity noise, due to the positive correlation between mode hopping effects and cavity length. Double resonance, which was achieved through the combined effect of a self-injection locking approach and the use of a single cut technique, was expected to allow for the cavity length to be reduced down to a few meters without significant losses in threshold seed pump power and maximum output. Experimental evaluation showed that the new cavity had a threshold seed pump power of $10 \mathrm{~mW}$, up from the $2 \mathrm{~mW}$ found in previous design, while having a maximum output power of $1.5 \mathrm{~mW}$, up from $0.5 \mathrm{~mW}$, showing how the single cut technique allowed for a threshold power of the same order of magnitude despite the great reduction of gain medium, while providing an even greater maximum output power. In terms of output linewidth, the short cavity and selfinjection locking were found to allow for a Stokes output with an extremely narrow linewidth of $10 \mathrm{kHz}$ from an original source of $350 \mathrm{kHz}$, down from the $2 \mathrm{MHz}$ linewidth found for long cavity Brillouin Ring Lasers. In addition, self-injection locking was found to significantly improve lasing stability, allowing for lasing intervals of up to $90 \mathrm{~ms}$, compared to the $10 \mathrm{~ms}$ ones obtainable without it. Finally, the intensity noise improvements provided by a short cavity, were evaluated through RIN measurements, obtaining values of $\sim-145 \mathrm{~dB} / \mathrm{Hz}$ across the whole $0-800 \mathrm{MHz}$ range, which were compared to
RIN measurements performed on previous long cavity designs, which reached significantly higher values, up to $-90 \mathrm{~dB} / \mathrm{Hz}$. Compared to the long cavity, the RIN improvements are shown to translate to a BOTDA strain and temperature resolution improvement by up to a factor of 3.5 .

From these results it can be seen how the stabilized doubly resonant BRL design can provide substantial improvements in a variety of applications. For what concerns BOTDA, it has the potential to be successfully employed as a pump-probe source in accurate Brillouin optical time-domain sensor systems.

This activity has received funding from the European Union through Horizon 2020, the Framework Programme for Research and Innovation, under project SLAM-DAST, Grant Agreement No. 971149.

\section{References}

1. S. P. Smith, F. Zarinetchi, S. Ezekiel, Opt. Lett., 16, 393-395 (1991)

2. P. A. Nicati, K. Toyama, S. Huang, H. J. Shaw, IEEE Photonics Technol. Lett., 6, 801-803 (1994).

3. M.A. Soto, P.K. Sahu, S. Faralli, G. Sacchi, G. Bolognini, F. Di Pasquale, B. Nebendahl, C. Rueck, Proceedings of SPIE, 66193B (2017)

4. D. Marini, L. Rossi, F. Bastianini, G. Bolognini, 20th Italian National Conference on Photonic Technologies (Fotonica 2018), CP748, 147646 (2018)

5. D. Marini, L. Rossi, F. Bastianini, G. Bolognini, OSA Technical Digest, ThE71, 140688 (2018).

6. L. Rossi, D. Marini, F. Bastianini, G. Bolognini, Opt. Express, 27, 29448-29460 (2019).

7. F. Falcetelli, L. Rossi, R. Di Sante, G. Bolognini, Sensors, 20, 3100 (2020)

8. M. Nikles, L. Thévenaz, P. A. Robert, Opt. Lett. 21, 758-760 (1996)

9. D. Marini, M. Iuliano, F. Bastianini and G. Bolognini, J. Light. Technol., 36, 1131-1137 (2018)

10. P. Nicati, K. Toyama, H.J. Shaw, J. Light. Technol., 13, 1445-1451 (1995).

11. V. V. Spirin, C. A. López-Mercado, S.I. Kablukov, E.A. Zlobina, I.O. Zolotovskiy, P. Mégret, A.A. Fotiadi, Opt. Lett., 38, 2528-2530 (2013)

12. P. Horak, W.H. Loh, Opt. Expr., 14, 3923-3928 (2006).

13. D. Derickson, Fiber optic test and measurement (Prentice Hall, 1998).

14. G. Bolognini, S. Faralli, A. Chiuchiarelli, F. Falconi, F. Di Pasquale, IEEE Photonics Technol. Lett., 18, 1591-1593 (2006).

15. Z. Ou, X. Bao, Y. Li, B. Saxena and L. Chen, IEEE Photonic. Tech. L., 26, 2058-2061 (2014).

16. M.A. Soto, G. Bolognini and F. Di Pasquale, Opt. Exp., 18, 14878-14892 (2010). 